\title{
ARCEBS in brief
}

\author{
Susanta Lahiri • S. K. Das
}

Received: 18 May 2011 / Published online: 3 June 2011

(C) Akadémiai Kiadó, Budapest, Hungary 2011

The second international conference on Application of Radiotracers in Chemical, Environmental and Biological Sciences (ARCEBS-10) was organized by the Saha Institute of Nuclear Physics (SINP) in cooperation with International Atomic Energy Agency (IAEA), and was held in the SINP premises during November 7-13, 2010 in Kolkata, India. From its very inception, "ARCEBS" series of conference attracted researchers from all over the globe from various disciplines of science with the common thread of radiotracers or energetic ion beams. The peer community considers ARCEBS series as platform for trans-disciplinary research and exchanging ideas, ARCEBS-10 is no exception from that. ARCEBS keeps an eye on the cutting edge developments of the world on accelerator based technologies and proposed large scale future facilities like EURISOL or FAIR. This is the reason we have included an article on FAIR written by

S. Lahiri $(\square)$

Saha Institute of Nuclear Physics, 1/AF Bidhannagar,

Kolkata 700064, India

e-mail: susanta.lahiri@saha.ac.in

S. K. Das

Variable Energy Cyclotron Centre, 1/AF Bidhannagar, Kolkata 700064, India
Professor Bikash Sinha, a renowned high energy physicist. By incorporating this article we would like to give the message that future generation nuclear chemists have lot to play with these kinds of huge radioisotope production facilities starting from the target development to the application of exotic radionuclides for the beneficial use of mankind.

The organization of a conference like ARCEBS-10 is a collective effort and charter of cooperation of many. It is our duty to acknowledge the people whose effort made the conference a grand success.

Professor Tibor Braun, Editor-in-Chief of the Journal of Radioanalytical and Nuclear Chemistry (JRNC) readily agreed to publish ARCEBS proceedings in a special issue of JRNC. Professor A. Chatt is the Editor-in-charge for conference proceedings of JRNC. His support helped us a lot to bring out this issue. The man behind the excellent electronic submission platform of JRNC articles is Dr. Andras Schubert. All the articles were published in the fastest possible time in the "on line first" of JRNC due to the effort of Dr. Schubert. We express our sincere thanks to Professors Tibor Braun, A. Chatt and Andras Schubert.

We are deeply indebted to Professor Milan K. Sanyal, Director, SINP, for his generous financial help to organize the conference and his encouragement and suggestions in every step of the conference. We are grateful to Dr. R. K. Bhandari, Director, Variable Energy Cyclotron Centre (VECC), for his cooperation towards organizing the conference both in the form of financial help and other logistic supports. We thankfully acknowledge the encouragement received from Professor Bikash Sinha, Chairman, International Advisory Committee of ARCEBS-10 and Ex-Director of SINP and VECC. Professor Sinha is always a source of inspiration to us. 


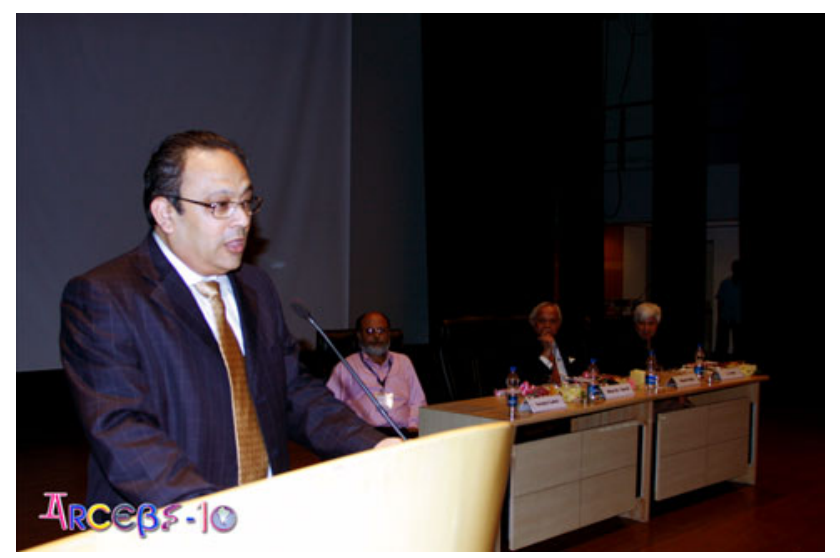

The inaugural programme of ARCEBS-10 is addressed by Professor Milan K. Sanyal, Director SINP

The Department of Atomic Energy, Govt. of India took keen interest in ARCEBS-10 for promoting application of radioisotopes for the betterment of life. Dr. Srikumar Banerjee, Chairman, Atomic Energy Commission, Govt. of India and Secretary, Department of Atomic Energy, Govt of India made it possible to come and deliver a lecture in ARCEBS-10 despite of his extremely busy schedule. We are grateful to him. We acknowledge the financial supports from the Board of Research in Nuclear Sciences (BRNS), the Council of Scientific and Industrial Research (CSIR), the Department of Science and Technology (DST), the Indian National Science Academy (INSA), and ThermoFischer Scientific, India. A big amount of money was spent from the DAE-SINP XIth 5 year plan project "Trace Analysis: Detection, Dynamics and Speciation (TADDS)".

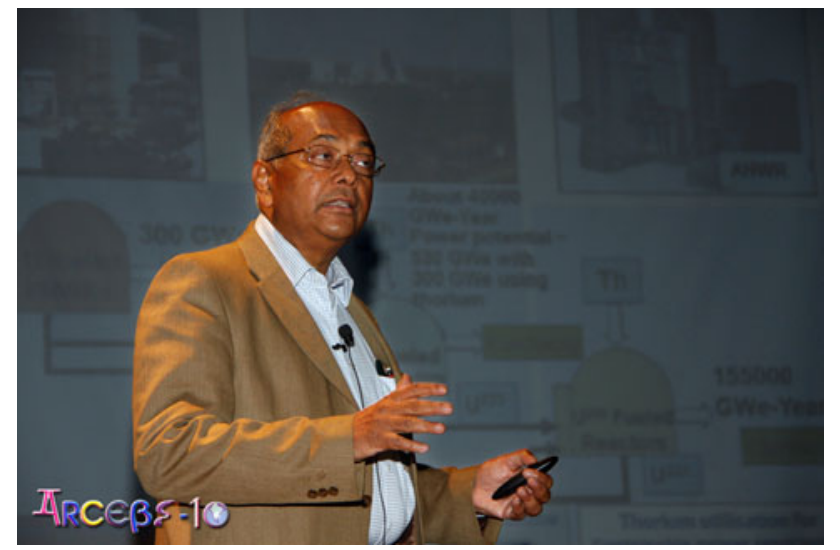

Dr. Srikumar Banerjee, Chairman, Atomic Energy Commission, and Secretary, Department of Atomic Energy, Govt. of India is addressing delegates of ARCEBS-10.

The success of a conference always depends on its technical content. The reviewers from all over the globe helped to maintain the standard of the scientific contribu- tion very high for the volume of the extended abstract and this special issue of JRNC. We wish to record our sincere appreciation to all the reviewers. We are also thankful to Professor N. R. Das, Chairman, Scientific Committee of ARCEBS-10 for his help and day to day interest in organizing ARCEBS-10. We are equally grateful to all the plenary speakers.

ARCEBS-10 allotted one full day for the presentations of young scientists, oral or poster, and bestowed prestigious awards to the young researchers for best oral and poster presentations. We are grateful to Professor Y. Nagame and Professor Andreas Türler, chairpersons of the judges panel for oral and poster presentation and the other members of these two committees for spending time for this purpose. Congratulation to the young awardees: Moumita Maiti, Sanhita Chaudhury, Ashok Behera (oral), and Binita Dutta, Seweryn Krajewski, Nisha Barot (poster).

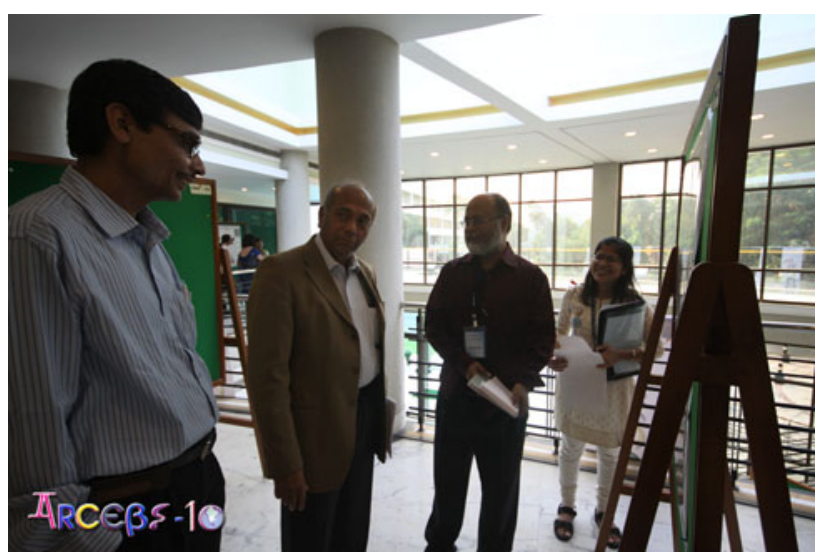

Dr. Srikumar Banerjee, Chairman, Atomic Energy Commission, Govt of India is in the poster session of ARCEBS-10

Professor N. R. Das on the occasion of ARCEBS-10 brought out a souvenir with life sketch of three pioneering scientists. In the souvenir he wrote an article on the life and dedication of Madame Curie. An article on George Hevesy written by Professor A. Vértes was reprinted from older issue of JRNC. We are grateful to Professor Vértes as well as to Professor Braun for extending permission to reprint the article from JRNC. Professor Atri Mukhopadhyay wrote an article on life and works of our founder director Professor Meghnad Saha. We are thankful to him.

We sincerely thank the members of local organizing committee, national organizing committee, scientific committee and international advisory board. We are thankful to the young research fellows of the radiochemistry group of SINP, Swadesh, Binita, Ajoy and Kaustub for their untiring efforts to make the conference a memorable one. 


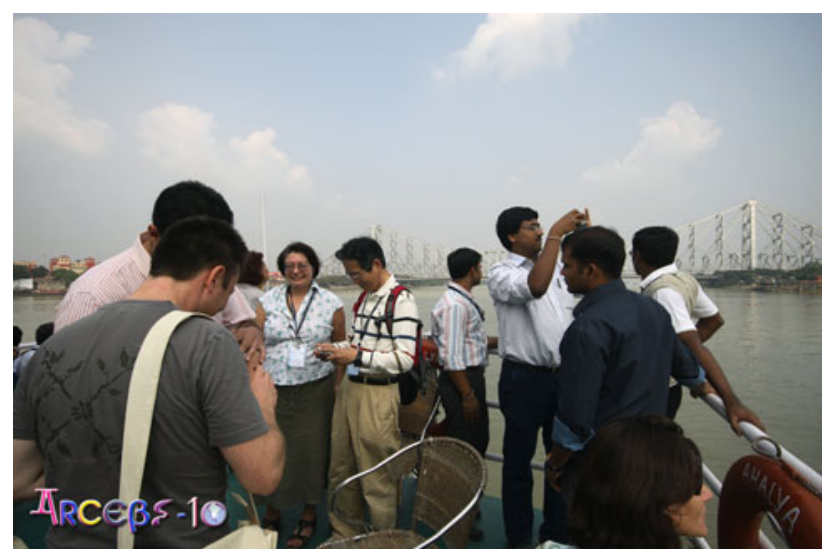

Participants of ARCEBS-10 are on a boat trip on the river Hooghly

Dr. Moumita Maiti was the convener, local organizing committee of ARCEBS-10, secretary, scientific committee, as well one of the editors of the extended abstract book of
ARCEBS-10. No word of appreciation is sufficient for her. Her intellectual and organization effort is one of the key pillar behind the success of ARCEBS-10.

ARCEBS is observing an interesting evolution, evolution towards the excellence, evolution towards more transdisciplinary research. ARCEBS is now not restricted to chemistry, environment and biology, but more and more contributions are coming from physics, material science, accelerator technology, nuclear medicine, geochronology, etc. Keeping admiration to this interdisciplinary quest, a new definition of ARCEBS may be more appropriate. We invite all of you to share with us the excitement, join us with new broader definition of ARCEBS, Application of RadiotraCers and Energetic Beams in Sciences in 2014.

We look forward to meet all of you again in ARCEBS2014. 